\title{
EVALUATION OF ENSEMBLE COHERENCE AS A MEASURE FOR STOCHASTIC AND SYSTEMATIC PHASE INCONSISTENCIES
}

\author{
Homa Ansari, Fernando Rodriguez Gonzalez, Ramon Brcic, Francesco De Zan \\ Remote Sensing Technology Institute (IMF), German Aerospace Center (DLR)
}

\begin{abstract}
The presence of stochastic and systematic inconsistencies is a concern for the precision and interpretability of Interferometric Synthetic Aperture Radar (InSAR) when distributed scatterers are exploited for SAR time series analysis [1]. Multitemporal phase estimators aim at retrieving a consistent common-master interferometric time series, thereby reducing the effect of stochastic inconsistencies. Exploiting data redundancy the latter estimators are theoretically expected to decrease the susceptibility to systematic inconsistencies as well. In this contribution we seek a computationally efficient quality measure to show the effectiveness of multitemporal phase estimation in the reduction of inconsistencies. Choosing the ensemble coherence as a candidate, we firstly seek a constant false alarm rate detector for initial detection of signal-bearing areas. Furthermore the impact of phase inconsistencies on the ensemble coherence will be brought into attention.
\end{abstract}

Index Terms - Distributed Scatterers, Estimation Efficiency, InSAR Time Series, Multipass SAR Interferometry, Phase Inconsistencies, Quality Measure

\section{INTRODUCTION}

Distributed Scatterer (DS) interferometry aims at the exploitation of statistically homogeneous and partially coherent areas within the Synthetic Aperture Radar (SAR) time series for deformation signal retrieval. In order to account for signal decorrelation within the time series, different phase estimators are proposed to improve the Signal to Noise Ratio (SNR) of DS phases $[2,3,4,5,6,1]$. The latter estimators exploit all possible noise-corrupted multi-master interferograms within a SAR time series, to retrieve a consistent common-master phase-series pertaining to the deformation and atmospheric signals. Following such common-master interferogram estimation, the interferometric phase inconsistencies within the SAR time series [7] are expected to be effectively reduced. The estimated interferogram time series is further utilized in the retrieval of the deformation signal [3]. Therefore, the precision and interpretability of these signals is driven, among other factors, by the quality of the estimated interferograms. Defining the latter quality as the variance and bias of the esti- mated phases, different measures may be defined to allow the detection and incorporation of high-precision DS phase-series into the deformation estimation.

Our focus in this work is to choose a computationally efficient quality measure and scrutinize its potential and limitations with respect to independent indicators of interferometric phase quality within the SAR time series. Evaluated a posteriori to phase estimation, the ensemble coherence [3] is among the most computationally efficient measures for evaluation of the quality of the retrieved DS phases. Therefore it is a potential candidate for Big InSAR Data processing [8]. In the following, we firstly introduce this measure and further describe the different methods to allow its investigation with respect to effective, albeit computationally expensive, quality indicators of the interferometric phases within a time series.

\section{THE ENSEMBLE COHERENCE}

Estimated a posteriori to the DS phase estimation, the ensemble coherence reads as:

$$
\hat{\gamma}=\frac{2}{n(n-1)} \sum_{i=1}^{n} \sum_{j=i+1}^{n} \cos \left(\Delta \phi_{i j}-\Delta \hat{\phi}_{i j}\right) ;
$$

where $n, \Delta \phi_{i j}$ and $\Delta \hat{\phi}_{i j}$, respectively indicate the number of images in the SAR time series, the observed (spatially multilooked over the DS region) interferometric phase and the estimated interferometric phase between the $i$ and $j$ SAR acquisitions in the time series. From equation (1), it is clear that $\hat{\gamma}$ reflects the unmodeled phases, but it disregards the statistical properties of the involved interferograms.

Defining closure phases as the phase mismatch among any arbitrary three interferograms pertaining to the DS region within the SAR time series, i.e.:

$$
\nabla \phi_{i j k}=\operatorname{wrap}\left\langle\Delta \phi_{i j}+\Delta \phi_{j k}+\Delta \phi_{k i}\right\rangle
$$

the DS phase is said to be inconsistent if the closure phase is nonzero. The underlying model in phase estimation assures the consistency of the estimated phase by imposing:

$$
\nabla \hat{\phi}_{i j k}=0
$$

for any combination of the estimated interferograms within the time series. 
(a)

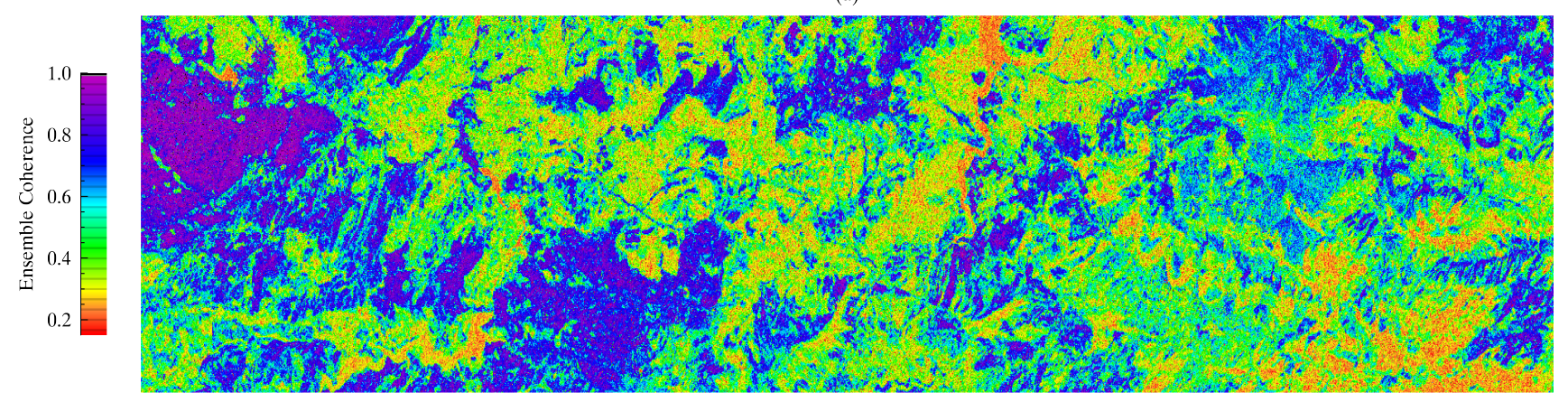

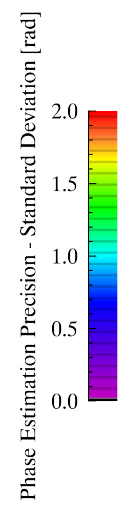

(b)

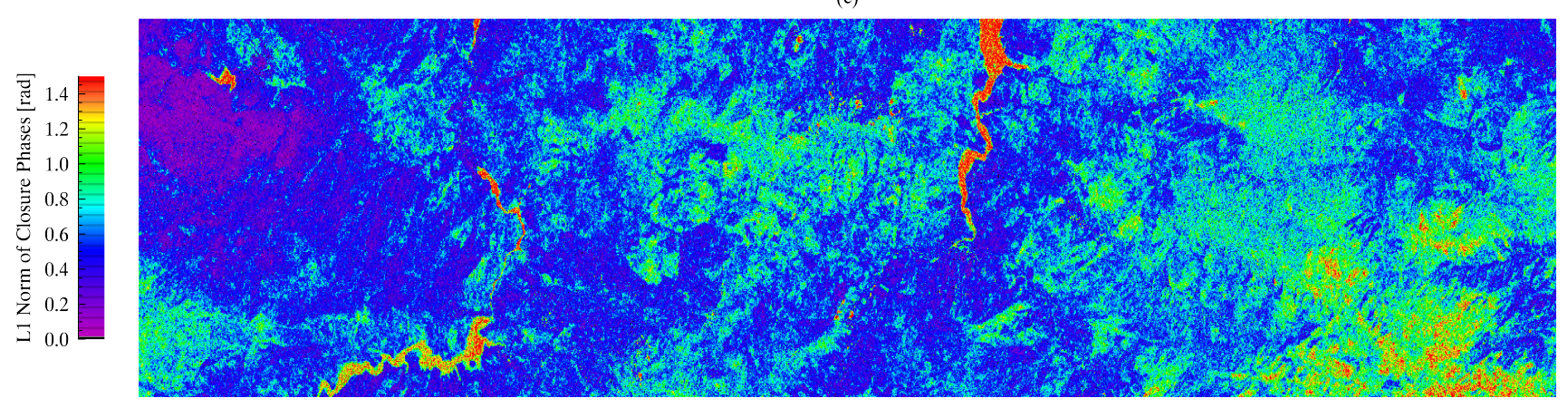

Fig. 1: Maps of different phase quality measures over a test site in Turkey using 35 Sentinel-1 acquisitions, (a) the ensemble coherence map evaluated after temporal phase estimation using EMI estimator [6]; (b) standard deviation of phase estimation for the interferogram with temporal baseline of 444 days, evaluated a posteriori to EMI phase estimation; (c) L1 norm of a subset of closure phases within the SAR time series, the subset refers to lag-1 interferometric phases i.e $\nabla \phi_{1,2,3}, \nabla \phi_{2,3,4}, \ldots, \nabla \phi_{33,34,35}$. Note that the estimation kernel is identical in (a) and (c) but it differs in (b), hence the lower resolution of the features in the latter map. A correlation between the three map is visually observed and further demonstrated in Fig 2.

With this definition of the phase estimation model, the residual phases as reflected in equation (1) shall theoretically contain the inconsistent phase within the time series. The latter may be categorized into stochastic and systematic effects [7]. For large ensemble sizes, the closure phase pertaining to stochastic inconsistencies asymptotically approaches zero. The systematic inconsistencies, on the other hand, indicate the presence of a physical inconsistent signal within the time series and the corresponding ensemble. It is attributed, for instance, to the variation of the dielectric properties of the subresolution scatterers between different acquisition times $[9,7]$. The presence of such physical effects complicates the interpretation of the target atmospheric and deformation signals and renders the susceptibility of the retrieved DS phases to estimation biases [7]. 


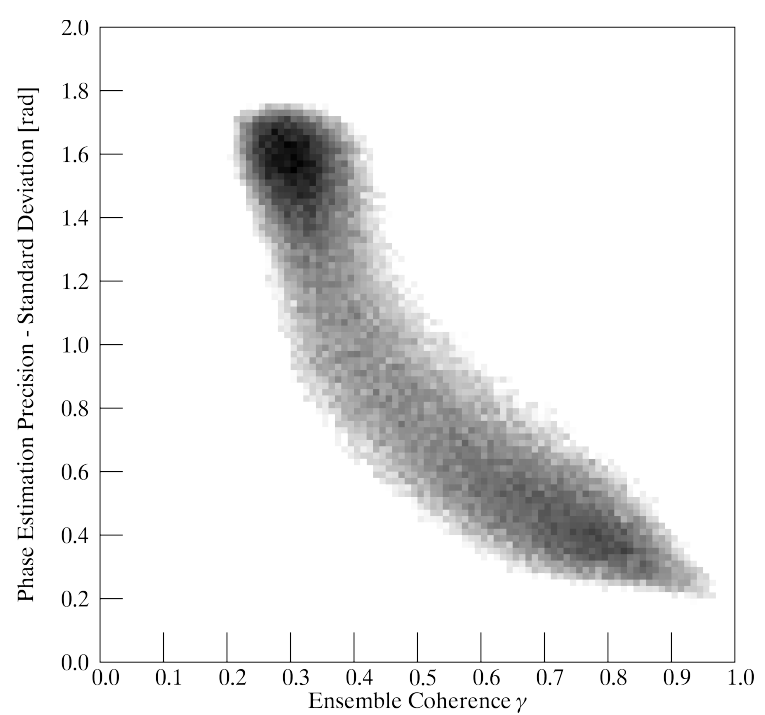

(a)

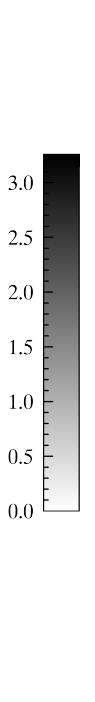

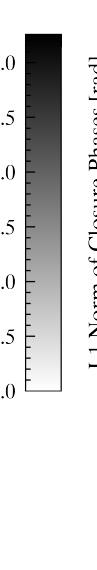

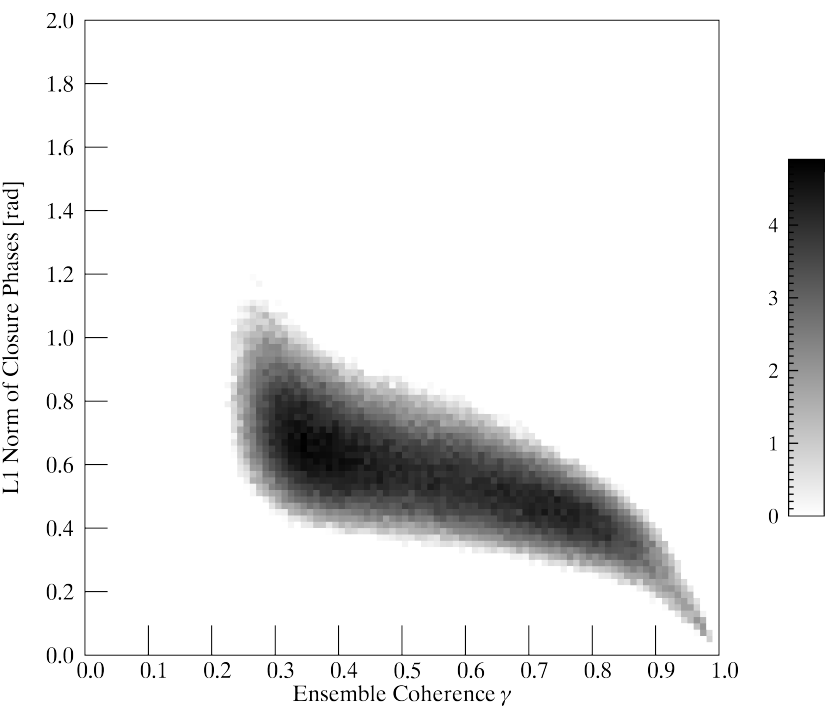

(b)

Fig. 2: Bivariate probability distribution of (a) the ensemble coherence and phase estimation precision and (b) the ensemble coherence and L1 norm of closure phases. The correlation between the measures reads as (a) $\rho_{a}=-0.71$ and (b) $\rho_{b}=-0.64$. For low coherence regions corresponding to $\hat{\gamma} \leq 0.3$, the compared quality measures are decorrelated $\left(\rho_{a}=0.01, \rho_{b}=-0.08\right)$. The latter threshold is resulted from our simulation analysis of pure clutter DS. The observed correlations indicate the impact of inconsistencies on ensemble coherence, the detection of systematic from the stochastic inconsistencies using the ensemble coherence is, however, subject to further research.

\section{IMPACT OF PHASE INCONSISTENCIES ON ENSEMBLE COHERENCE}

Phase estimation is designed for reducing the stochastic inconsistencies by exploiting the temporal data redundancy [3]. Exploiting this redundancy it may as well reduce the susceptibility of the estimated interferograms to systematic inconsistencies [7]. The impact of stochastic and systematic phase inconsistencies on the ensemble coherence is evaluated from the following aspects:

The initial DS candidate detection using the ensemble coherence is investigated. Evaluating the Probability Density Function (PDF) of the measure for pure clutter, a constant false alarm rate detector is sought for detecting signal-bearing DSs. The latter allows for an objective selection of a detection threshold for inclusion of DSs into deformation signal analysis.

The capability of ensemble coherence in showing the effectiveness of phase estimation in reducing the phase noise shall be scrutinized. For this investigation, an independent measure of phase estimation variance is taken as the benchmark $[6,1]$. Under the assumption of spatial stationarity of the physical signals within an estimation kernel, the spatial phase variance is suggested as a proxy for the evaluation of the a posteriori phase estimation variance $[6,1]$. Here we evaluate the correlation between the ensemble coherence and the latter phase variance using a test case with Sentinel-1 time series comprising of 35 acquisitions over Turkey (see Fig. 1.(a) and (b) and 2.(a) for the preliminary results). Further simulation and in-depth analysis with wide area processing is in progress and will be presented.

The potential and limitation of ensemble coherence in reflecting the phase inconsistencies is a related topic worthy of investigation. Two measures are introduced as the indicators of the latter inconsistencies based on the observed closure phases within the data stack, i.e. the L1 norm of the closure phases [7] and a chi-squared distributed statistical measure [10]. The performance bottleneck as well as computational cost of both approaches lies in the number of investigated closure phases. The relation of the ensemble coherence to these effective measures of inconsistencies is studied using the same time series of Sentinel-1 data over Turkey (see Fig. 1.(a) and (c) and 2.(b) for the preliminary results). Further simulation and in-depth analysis with wide area processing is in progress and will be presented.

The detection of systematic from the stochastic inconsistencies is of importance for safeguarding against the interfering signals in deformation analysis. This topic has been investigated in [10] through the proposal of a chi-squared distributed statistical measure. The latter incorporates the closure phases along with their stochastic properties. The robustness of this measure is however gained at the cost of increased computational effort; furthermore the proposed measure is sensitive to the inevitable errors in the second order statistics of the closure phases [10]. 


\section{SUMMARY AND OUTLOOK}

Estimated a posteriori to temporal phase estimation for interferogram stacks, the ensemble coherence is a computationally efficient quality measure for the integration of high-precision DSs into deformation analysis. The performance of this measure in reflecting the quality of the estimated phases shall however be investigated. We show the correlation between the independent measures of phase quality with the ensemble coherence and propose a constant false alarm rate detector for inclusion of DSs into signal retrieval. Further in-depth analysis with simulation studies and wide area data processing is in progress and will be presented.

The ensemble coherence does not regard the stochastic model of the data in its evaluation. The proposal of computationally efficient quality measures which allow for the inclusion of the stochastic model is a relevant research topic. The detection of the systematic from the stochastic inconsistencies using only the ensemble coherence is subject to further investigations.

\section{REFERENCES}

[1] Homa Ansari, Efficient High-Precision Time Series Analysis for Synthetic Aperture Radar Interferometry, $\mathrm{Ph} . \mathrm{D}$. thesis, German Aerospace Center and Technical Univercity of Munich, 2018.

[2] Andrea Monti Guarnieri and Stefano Tebaldini, "On the Exploitation of Target Statistics for SAR Interferometry Applications," IEEE Transactions on Geoscience and Remote Sensing, vol. 46, no. 11, pp. 3436-3443, Nov. 2008.

[3] Alessandro Ferretti, Alfio Fumagalli, Fabrizio Novali, Claudio Prati, Fabio Rocca, and Alessio Rucci, "A New Algorithm for Processing Interferometric Data-Stacks: SqueeSAR," IEEE Transactions on Geoscience and Remote Sensing, vol. 49, no. 9, pp. 3460-3470, Sept. 2011.

[4] F De Zan, F Rocca, and A Rucci, "PS processing with decorrelating targets," in Proc. Envisat Symp., 2007, pp. $1-5$.

[5] Gianfranco Fornaro, Simona Verde, Diego Reale, and Antonio Pauciullo, "CAESAR: An Approach Based on Covariance Matrix Decomposition to Improve Multibaseline \& Multitemporal Interferometric SAR Processing," IEEE Transactions on Geoscience and Remote Sensing, vol. 53, no. 4, pp. 2050-2065, Apr. 2015.

[6] Homa Ansari, Francesco De Zan, and Richard Bamler, "Efficient Phase Estimation for Interferogram Stacks," IEEE Transactions on Geoscience and Remote Sensing, vol. 56, no. 7, pp. 4109-4125, May 2018.
[7] Francesco De Zan, Mariantonietta Zonno, and Paco Lopez-Dekker, "Phase Inconsistencies and Multiple Scattering in SAR Interferometry," IEEE Transactions on Geoscience and Remote Sensing, vol. 53, no. 12, pp. 6608-6616, Dec. 2015.

[8] Homa Ansari, Francesco De Zan, and Richard Bamler, "Sequential Estimator: Toward Efficient InSAR Time Series Analysis," IEEE Transactions on Geoscience and Remote Sensing, vol. 55, no. 10, pp. 5637-5652, Oct. 2017.

[9] Francesco De Zan, Alessandro Parizzi, Pau Prats-Iraola, and Paco López-Dekker, "A SAR interferometric model for soil moisture," IEEE Transactions on Geoscience and Remote Sensing, vol. 52, no. 1, pp. 418-425, 2014.

[10] Simon Zwieback, Xingyu Liu, Sofia Antonova, Birgit Heim, Annett Bartsch, Julia Boike, and Irena Hajnsek, "A Statistical Test of Phase Closure to Detect Influences on DInSAR Deformation Estimates Besides Displacements and Decorrelation Noise: Two Case Studies in High-Latitude Regions," IEEE Transactions on Geoscience and Remote Sensing, vol. 54, no. 9, pp. 55885601, Sept. 2016. 